\title{
Case Report of Surgical Excision of Non-Communicating Rudimentary Horn of Uterus Present in Adolescent with Dysmenorrhea
}

\author{
Majed Alshahrani \\ Department of Obstetrics and Gynecology, College of Medicine, Najran University, Najran, \\ Kingdom of Saudi Arabia \\ Email: alkozeem@hotmail.com
}

Received 9 July 2015; accepted 18 August 2015; published 21 August 2015

Copyright (C) 2015 by author and Scientific Research Publishing Inc.

This work is licensed under the Creative Commons Attribution-NonCommercial International License (CC BY-NC).

http://creativecommons.org/licenses/by-nc/4.0/

(c) (7) (2) Open Access

\begin{abstract}
Unicornuate uterus with a rudimentary horn is a rare type of müllerian duct malformation. It leads to many obstetrical and gynecological complications. The mean age of presentation was the mid $20 \mathrm{~s}$ [1]. It is one of the differential diagnosis of dysmenorrhea in adolescent. A 19-year-old single was present with progressive severe dysmenorrhea and chronic pelvic pain after history right ovarian torsion treated by right salpinoophrectomy. Exploratory laparotomy was performed found unicornuate uterus with right non-communication rudimentary horn. Excised of right rudimentary horn was done. Remove of non-communication rudimentary horn will reduce symptoms of dysmenorrhea and chronic pelvic pain. Proper diagnosis and management will prevent misdiagnosis and good outcome to the patient.
\end{abstract}

\section{Keywords}

Rudimentary Horn, Dysmenorrhea, Surgical Excised

\section{Introduction}

The female reproductive organs develop at approximately the sixth week of gestation from the paired müllerian (paramesonephric) ducts, which fuse to create the uterus, cervix, and upper two-thirds of the vagina. Failure of fusion or normal development or incomplete medial wall resorption of the müllerian ducts can result in a variety 
of congenital uterine anomalies.

Unicornuate uterus with a rudimentary horn is a rare type of müllerian duct malformation. The unicornuate uterus is a result of abnormal or failed development of one of the paired müllerian ducts. Unicornuate uterus accounts for approximately $3 \%-13 \%$ of all müllerian anomalies [2] [3].

The American Fertility Society classification divides Unicornuate uterus into 4 categories: 1) unicornuate uterus with a communicating rudimentary horn, 2) unicornuate uterus with a non-communicating rudimentary horn, 3) unicornuate uterus with rudimentary horn without cavity and 4) isolated unicornuate uterus [4].

The rudimentary horn could be functional endometrium or not. It can lead to many obstetrical and gynecological complications. Besides gynecologic complications, such as endometriosis, dysmenorrhea, primary infertility, and hematometra, anomalies of the urinary system and obstetrical problems, such as malpresentation, miscarriages, and premature birth and ectopic pregnancy [5].

This report explained that the proper diagnosis and treatment of a patient with unicornuate uterus with a non-communicating rudimentary horn who had history of salpingoopherectomy was aggravated her symptoms of dysmenorrhea. Surgical resection non-communicating rudimentary horn was resolved her dysmenorrhea.

\section{Case Report}

The patient is a 19-year-old, unmarried. Patient was admitted to Abha General Hospital in Obstetrics and Gynecology department. Patient had history of emergency laparotomy at age of 16 years for right ovarian torsion and treated by right salpingoopherectomy. Then patient started to complain from progressive cyclic dysmenorrhea and chronic pelvic pain not response to analgesia for several times. After that patient was placed on combined pill for several month but still complain from dysmenorrhea. Exploratory laparotomy was performed by pfannenstiel incision. Inter to peritoneal cavity were the right non-communicating rudimentary horn attached to uterus. The scar of previous surgery was seen in right side of rudimentary horn. The uterus was normal with normal position fallopian tube and ovary (Figure 1). Decision was made for excised the right rudimentary horn by cutting and dissected it and sutured uterus part with maintain hemostaiss (Figure 2). Cross-section of rudimentary horn showed functional endometrial with material inside (Figure 3). The patient was discharged day 3 postoperative in good condition. The patient attended outpatient clinic after 4 month for follow up with resolved the symptom of dysmenorrhea.

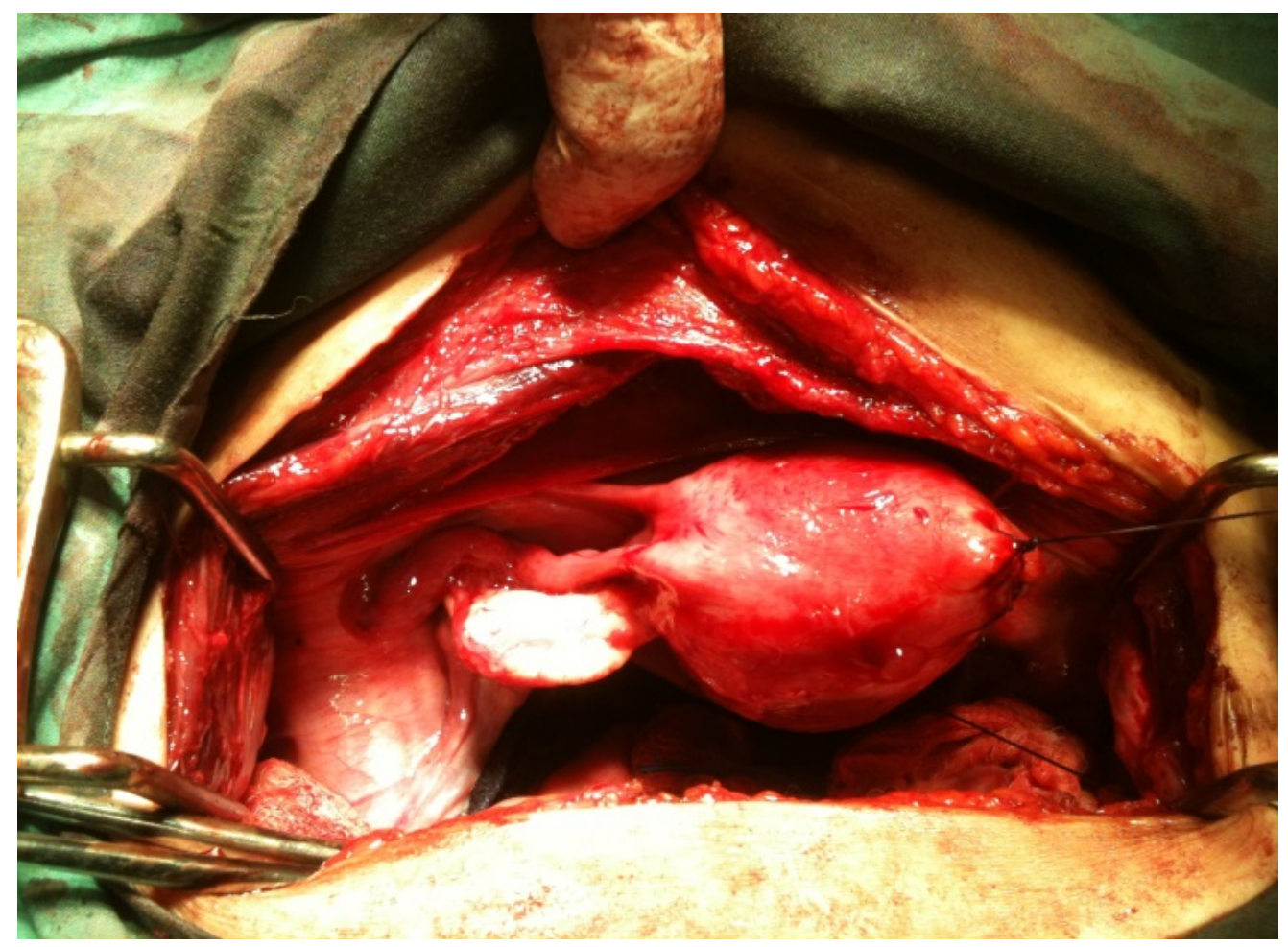

Figure 1. Normal left ovary and fallopion tube. 


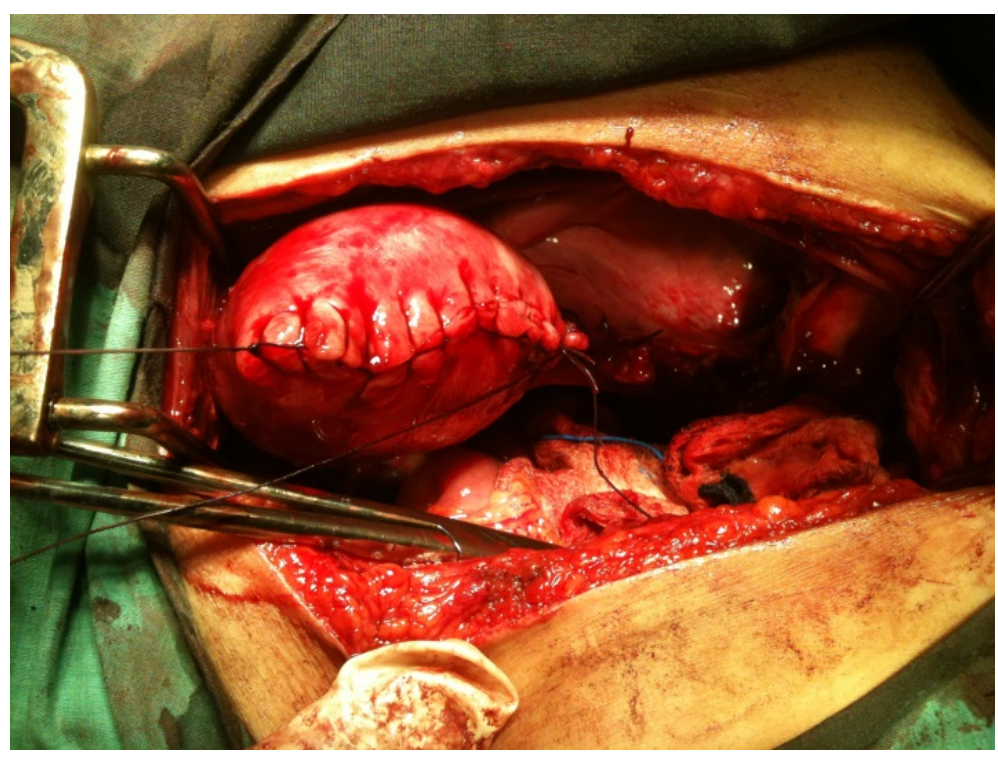

Figure 2. Sutured part of uterus after excised.

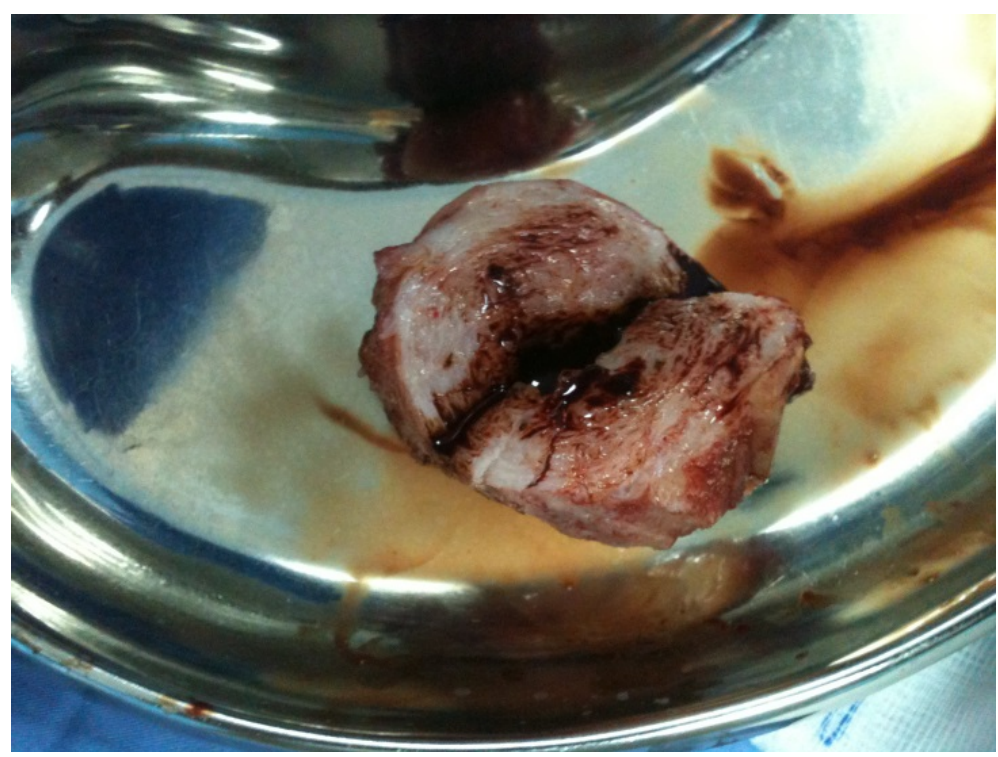

Figure 3. Cross-section of rudimentary horn.

\section{Discussion}

The prevalence of dysmenorrhea is estimated to be around $25 \%$ of all women and $90 \%$ of adolescent [6]. The rudimentary horn may content functional endometrial cavity or not. Non-communicating rudimentary horns that have an endometrial cavity are the most common unicornuate subtype and are the most clinically complication.

Women with unicornuate uterus with a non-communicating rudimentary horn usually are present with progressive dysmenorrhea and pelvic pain. The presentation can be delayed with increasing severity of dysmenorrhea, with each subsequent menstrual period as a common feature. The symptoms will interfere with quality of life and may not respond to medication like this patient. The correct diagnosis and proper management will prevent all the complication to the patient. This patient was missed diagnosis during emergency laparotomy lead progressive dysmenorrhea.

The diagnosis of uterine anomalies is difficult in some cases. It needs non-invasive diagnostic method, such as MRI before any intervention procedure and for looking for other anomalies associated with it. MRI is a good 
method for differentiation of all the different type of uterine anomaly. The MRI was not done because MRI was not available at that time of the presenting patient. This patient was presented with dysmenorrhea after salpingoopherectomy which caused obstruction of outflow of menstrual blood in functional non-communicating horn which was not improved by medication.

Surgical removal of the non-communicating horn is commonly performed to prevent complication of obstetrics and gynecology such as ectopic pregnancy, rupture rudimentary horn, endometriosis, preterm labour and to improve the symptoms of dysmenorrhea and chronic pelvic pain of the patient.

In conclusion, I present this case of laparotomy resection of a rudimentary uterine horn in a patient with progressive dysmenorrhea which symptoms are progressive post right salpingoopherectomy, which leads to blocked retrograde menstruation. Care must be taken to diagnose uterine anomaly and proper management should be conducted before intervention and it should be noted that misdiagnosis can severely impact a patient's quality of life.

\section{References}

[1] Jayasinghe, Y., Rane, A., Stalewski, H. and Grover, S. (2005) The Presentation and Early Diagnosis of the Rudimentary Uterine Horn. Obstetrics \& Gynecology, 105, 1456-1467. http://dx.doi.org/10.1097/01.AOG.0000161321.94364.56

[2] Grimbizis, G.F., Camus, M., Tarlatzis, B.C., Bontis, J.N. and Devroey, P. (2001) Clinical Implications of Uterine Malformations and Hysteroscopic Treatment Results. Human Reproduction Update, 7, 161-174. http://dx.doi.org/10.1093/humupd/7.2.161

[3] Acién, P. (1997) Incidence of Müllerian Defects in Fertile and Infertile Women. Human Reproduction, 12, $1372-1376$. http://dx.doi.org/10.1093/oxfordjournals.humrep.a019588

[4] The American Fertility Society (1988) The American Fertility Society Classifications of Adnexal Adhesions, Distal Tubal Occlusion, Secondary to Tubal Ligation, Tubal Pregnancies, Mullerian Anomalies and Intrauterine Adhesions. Fertility and Sterility, 49, 944-955.

[5] Kuscu, N.K., Lacin, S. and Kartal, O. (2002) Rupture of Rudimentary Horn Pregnancy at the 15th Week of Gestation: A Case Report. The European Journal of Obstetrics \& Gynecology and Reproductive Biology, 102, 209-210. http://dx.doi.org/10.1016/S0301-2115(01)00600-5

[6] Durain, D. (2004) Primary Dysmenorrhea; Assessment and Management Update. Journal of Midwifery \& Women's Health, 49, 520-528. http://dx.doi.org/10.1016/j.jmwh.2004.08.013 